\title{
Protocolo médico de reanimación cardiopulmonar asistida en casos de muerte súbita, una realidad
}

\author{
Sánchez-Arreola Luis Daniel1,2,3* \\ ${ }^{1}$ Subdirección Médica, Servicio de Urgencias, Hospital General La Villa, Secretaria de Salud; 2Jefatura de urgencias Hospital San Angel Inn \\ Chapultepec; ${ }^{3}$ Asesor Médico del Sistema de Atención de Emergencias 9-1-1, Centro Nacional de Información. Ciudad de México, México
}

\begin{abstract}
Resumen
Las enfermedades cardiovasculares en la actualidad son consideradas la principal causa de muerte en adultos de mediana edad en el mundo. Un gran porcentaje de estas muertes ocurre de forma súbita y en lugares o espacios públicos fuera del hospital. La supervivencia de una persona que sufre un paro cardíaco extrahospitalario es variable y está relacionada con ciertas determinantes como el inicio temprano de maniobras de reanimación cardiopulmonar (RCP) y desfibrilación precoz efectuadas por personal entrenado y realizadas dentro de los primeros 5 min de ocurrido el paro cardiorrespiratorio, asegurando así una mayor supervivencia y recuperación neurológica. Para lograr su efectividad, estas acciones deben ser realizadas por testigos en la escena del evento que cuenten con entrenamiento previo o mediante la asistencia de un operador telefónico de emergencia. En el presente artículo se describen los atributos del protocolo médico de reanimación cardiopulmonar asistida elaborado para operadores de emergencia del servicio de atención de llamadas de emergencia 9-1-1 elaborado en conjunto con el Instituto Mexicano del Seguro Social que está siendo ya utilizado en los 195 centros de emergencia que conforman el servicio nacional de atención de llamadas de emergencia en México.
\end{abstract}

Palabras clave: Reanimación cardiopulmonar asistida. RCP. Muerte súbita. 9-1-1. Operadores de emergencia. Protocolo médico.

\section{Medical dispatch protocol of assisted cardiopulmonary resuscitation in cases of sudden cardiac arrest, a reality}

\section{Abstract}

Cardiovascular disease is currently considered the leading cause of death in middle-aged adults all over the world. A large percentage of sudden deaths occur suddenly and in public places outside the hospital. Survival after out-of-hospital cardiac arrest is variable and is related with several conditions such as the early start of cardiopulmonary resuscitation maneuvers (CPR) by trained or telephone assisted personnel and the access to early defibrillation; both procedures must be performed within the first 5 min after sudden cardiac arrest has occurred to ensure a longer survival and neurological recovery. These actions must be performed in the medical scene by witnesses with prior training or through the assistance of an emergency dispatcher. The present article describes the attributes of a medical dispatch protocol of CPR attended by emergency

\section{Correspondencia:}

*Sánchez-Arreola Luis Daniel

Gregorio Gelati, 101

Fecha de recepción: 22-02-2019 Fecha de aceptación: 13-03-2019 C.P. 11850, Ciudad de México, México E-mail: dano23sa@ @otmail.com DOI: 10.24875/REIE.19000019
Disponible en internet: 20-06-2019 Rev Educ Investig Emer. 2019;1(2):42-46 www.medicinadeemergencias.com 2604-6520 @ 2019 Sociedad Mexicana de Medicina de Emergencias, AC. Publicado por Permanyer México SA de CV. Este es un artículo Open Access bajo la licencia CC BY-NC-ND (http://creativecommons.org/licenses/by-nc-nd/4.0/). 
dispatchers developed in conjunction with the Mexican Social Security Institute that is being used by 195 emergency call centers for the Mexican National Call Emergency Service 9-1-1.

Key words: Assisted cardiopulmonary resuscitation. CPR. Sudden cardiac arrest. 9-1-1. Emergency dispatchers. Medical protocol.

¿9-1-1, cuál es su emergencia?

Auxilio mi compañero de trabajo, que no se mueve y no

respira.

Conserve la calma, deme su ubicación y escuche con

atención...

Las enfermedades cardiovasculares en la actualidad son consideradas la principal causa de muerte en adultos de mediana edad en el mundo. Un gran porcentaje de estas muertes ocurre de forma súbita y en lugares o espacios públicos fuera del hospital. La mayoría de los casos de muerte súbita se relacionan con personas que padecen afecciones o padecimientos cardíacos previamente diagnosticados, siendo el más común de todos la cardiopatía isquémica asociada además a múltiples factores de riesgo bien establecidos como son la obesidad, el sedentarismo, el tabaquismo, la hipertensión arterial y la diabetes mellitus, descritos en numerosos estudios clínicos actuales ${ }^{1,2}$. Desafortunadamente, la mayoría de estos casos ocurren fuera del hospital y preferentemente en lugares públicos, en los cuales por simple probabilidad se concentra una cantidad considerable de personas, tal es el caso de plazas públicas, centros comerciales, estadios y restaurantes, entre otros, en los cuales la persona que presenta muerte súbita empieza generalmente con un episodio de paro cardiorrespiratorio como primera y única manifestación de enfermedad y con un pronóstico sombrío y desafortunado ${ }^{3,4}$.

De acuerdo a Rodríguez Reyes, et al. (2015), hasta la fecha en México no existe un registro preciso de la incidencia de muertes por esta causa en comparación con otros países, como por ejemplo en EE.UU., en donde se reporta una incidencia de 70 a 155 casos por 100,000 habitantes/año, Francia, con 32.1 casos por millón de habitantes/año, o China, con 41.3 casos por 100,000 habitantes/año. Sin embargo, estadísticas publicadas en el año 2008 reportaron alrededor de 9,579 fallecimientos en adultos a consecuencia de cardiopatía isquémica, de los cuales al menos un 30\% se presentaron de forma súbita, lo que representa una incidencia estimada de 17,873 casos/año, cifra que en la actualidad seguramente será mucho mayor ${ }^{5}$.

Aunque la supervivencia de una persona que sufre un paro cardíaco súbito fuera del hospital es variable, se sabe que esta se relaciona con el inicio temprano de maniobras de reanimación cardiopulmonar (RCP), realizadas por personal entrenado ya sea de manera presencial o mediante la asistencia por vía telefónica de un operador de emergencia, así como el acceso a una desfibrilación precoz realizada con un desfibrilador automático externo (DAE), evidenciándose un mejor pronóstico cuando ambos procedimientos son realizados dentro de los primeros 5 min de ocurrido el paro cardiorrespiratorio súbito, con lo que se logra una supervivencia evaluada al alta hospitalaria mayor al $50 \%$ y con una buena recuperación neurológica. Para lograr su eficiencia, estas acciones deben ser realizadas por testigos en la escena que cuenten con entrenamiento previo o que sean auxiliados por un operador telefónico de emergencia mediante el seguimiento de un protocolo médico previamente autorizado y definido ${ }^{6}$.

Estas acciones han sido ampliamente descritas y representadas gráficamente en la llamada "cadena de la supervivencia» (Fig. 1) descrita por primera vez en las Guías de Soporte Vital Básico y Avanzado de la Asociación Americana del Corazón (AHA), por sus siglas en inglés en las cuales se mencionan como las intervenciones críticas necesarias que conforman el proceso integral de atención de un paciente que sufre un episodio de paro cardiorrespiratorio: reconocimiento precoz de la emergencia y activación de los servicios de emergencia prehospitalarios (SEM), RCP efectiva realizada por testigos, acceso a la desfibrilación temprana utilizando un DAE en un tiempo preferentemente menor a 5 minutos posterior al paro, soporte vital avanzado y cuidados posreanimación efectuados por personal profesional capacitado. Se denotan y enfatizan los primeros dos eslabones, ya que estos adquieren una importancia especial por ser aquellos que determinan el acceso al sistema de emergencias y la identificación precoz del evento, así como el inicio temprano del tratamiento efectuado por personal no necesariamente especializado. Estas acciones en conjunto determinan un mejor pronóstico y una mayor supervivencia.

Actualizaciones recientes del año 2018 a la última versión de las Guías de soporte vital básico y reanimación cardiopulmonar de calidad de la AHA hacen referencia por primera vez a un nivel de evidencia y recomendación determinados, denotando la importancia de la asistencia en tiempo real sobre la técnica de la RCP por parte del operador telefónico hacia los testigos 


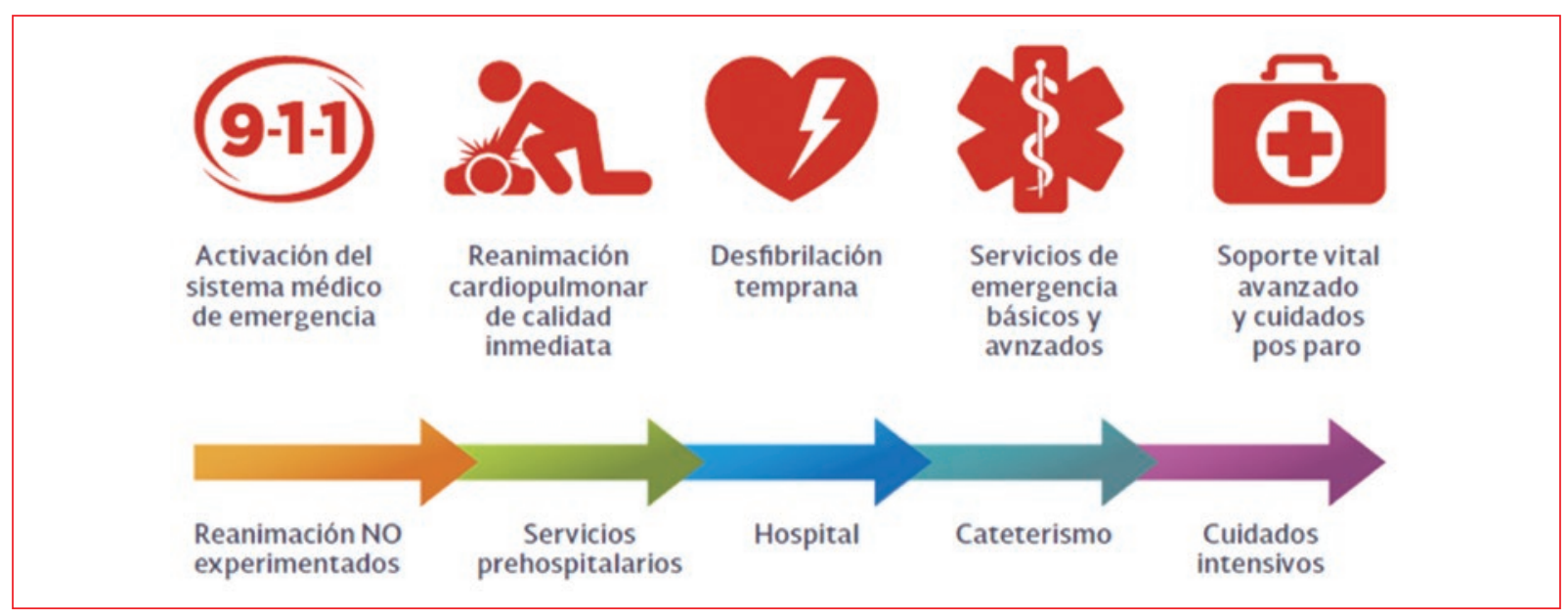

Figura 1. Cadena de la supervivencia, soporte cardiovascular básico (adaptada de Guías AHA para RCP y ACE 2015. Manual de capacitación para operadores telefónicos del 9-1-1 CNI. SESNSP).

Tabla 1. Recomendaciones para la reanimación cardiopulmonar (RCP) asistida por operadores telefónicos de emergencia en casos de paro cardiorrespiratorio extrahospitalario

Reanimación cardiopulmonar asistida, evidencias y recomendaciones 2018

Recomendación

Nivel de evidencia

Se recomienda que en casos de sospecha o confirmación de paro cardiorrespiratorio, el operador telefónico asista siempre al testigo en la escena sobre la técnica y maniobras de RCP básica

Clase I

Nivel de evidencia C-LD

En el caso de contar con testigos sin capacitación en soporte vital básico, en casos de paro

Clase I

cardiorrespiratorio el operador deberá asistir al testigo para que este inicie compresiones torácicas lo más

pronto posible con la técnica "solo con las manos"

Actualización 2017 AHA Guidelines for BLS and CPR Quality.

presentes en una situación de paro cardiorrespiratorio en el ambiente extrahospitalario. Estas recomendaciones se describen en la Tabla $1^{1}$.

Derivado de estas nuevas recomendaciones, diversos sistemas locales de emergencia en los EE.UU., Canadá y países del continente europeo han optado por dar mayor importancia a la implementación de protocolos médicos destinados a los operadores de emergencia para asistir a testigos en situaciones de paro cardiorrespiratorio extrahospitalario mostrando hasta ahora resultados favorables ${ }^{8,9}$. En el caso de México, derivado de la implementación del Servicio de Atención de Llamadas de Emergencia 9-1-1 en el año 2017 por decreto presidencial y gracias al trabajo conjunto entre la Secretaría de Salud, la Unidad de Educación, Investigación y Políticas de Salud del IMSS y la Sociedad Mexicana de Medicina de Emergencias, fue posible elaborar un protocolo médico telefónico destinado para que los operadores de emergencia brinden asistencia a los usuarios de las más de 40 mil llamadas que recibe el servicio anualmente a través del número de emergencias 9-1-1, las cuales son relacionadas con el reporte de probables víctimas de muerte súbita y que son efectuadas por medio de testigos en la escena. Además de la incorporación de este protocolo médico, fue posible realizar un trabajo que inició en el año 2017, destinado a rediseñar el «Protocolo General de Atención de la Llamada» utilizado por los operadores del 9-1-1, en el cual se agregó a la secuencia de la atención de la llamada una secuencia de acciones denominado «Esquema diferenciado de atención» con la finalidad de contar con un procedimiento alterno para la detección temprana de pacientes en paro cardiorrespiratorio basado en el reporte de los llamados «signos o focos rojos" (no está alerta, no responde, no se mueve), signos relacionados con una alta sospecha de tratarse de un paciente en paro cardiorrespiratorio. Este esquema alterno de atención favorece que el operador telefónico abrevie el interrogatorio inicial, acelerando la toma de datos primarios de contacto y dando prioridad al despacho casi simultáneo de una ambulancia en un tiempo no mayor a los 40 segundos 


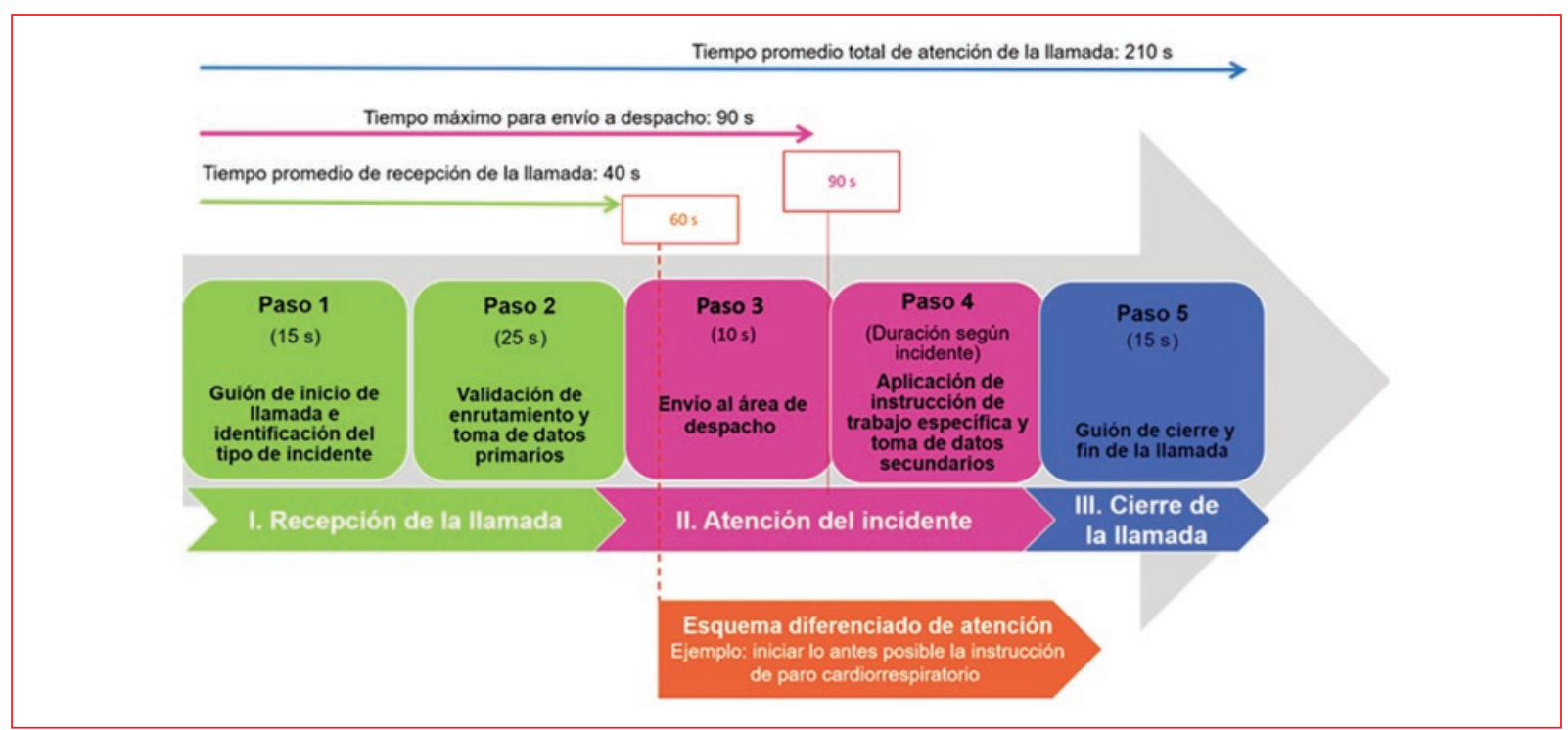

Figura 2. Esquema general. Protocolo General de Atención de la Llamada. Servicio de Atención de Llamadas de Emergencia 9-1-1.

CNI: Centro Nacional de Información; SESNSP: Secretariado Ejecutivo del Sistema Nacional de Seguridad Pública. 2017.

de haber iniciado la llamada, teniendo así oportunidad de iniciar cuanto antes la asesoría telefónica en maniobras de RCP básica al testigo en un tiempo total no mayor a los 60 segundos. Como parte complementaria, este esquema de atención obliga al operador a notificar a su supervisor en turno sobre el evento en curso con la finalidad de dar seguimiento estrecho a la llamada catalogándola como «llamada alta prioridad» y terminar la asistencia telefónica hasta confirmar la llegada de los SEM a la escena (Fig. 2).

Como parte de la elaboración del protocolo médico de asistencia telefónica para casos de paro cardiorrespiratorio extrahospitalario, se creó un modelo de gestión de calidad diseñado para cumplir determinados estándares e indicadores entre los que destacan el reconocimiento del paro cardiorrespiratorio por vía telefónica en al menos 1 minuto, el inicio de maniobras de reanimación a la víctima en no más de 60 segundos, el mantenimiento de las compresiones torácicas de forma continua y sin distracciones durante al menos 2 minutos $y$, por último, la realización de RCP básica para reanimadores legos con la técnica "solo con las manos» hasta la llegada del SEM, aspecto destacado en el apartado de soporte vital básico recomendado en las Guías de la AHA y del International Liaison Commitee on Resuscitation (ILCOR) desde el 2017 (Tabla 2) ${ }^{10}$.

Por último, y relacionado a la estructura del protocolo, es importante mencionar que este fue diseñado con la finalidad de que además de realizar las preguntas necesarias para obtener los datos de contacto y ubicación
Tabla 2. Estándares mínimos para la aplicación de reanimación cardiopulmonar de calidad

\begin{tabular}{|l|l|}
\hline $\begin{array}{l}\text { Estándares minimos de aplicación para considerar una } \\
\text { reanimación cardiopulmonar asistida adecuada }\end{array}$ \\
\hline Primero & $\begin{array}{l}\text { Reconocer un paro cardiorrespiratorio en el } \\
\text { 95\%de los casos en donde se reporta ausencia de } \\
\text { respuesta y respiración. }\end{array}$ \\
\hline Segundo & $\begin{array}{l}\text { Identificar un paro cardiorrespiratorio el minuto } \\
\text { desde que se recibe la llamada al sistema 9-1-1. }\end{array}$ \\
\hline Tercero & $\begin{array}{l}\text { Iniciar compresiones torácicas por parte del } \\
\text { testigo en el } 75 \% \text { de los casos en donde ésta no } \\
\text { haya sido inicida al momento de la llamada. }\end{array}$ \\
\hline Cuarto & $\begin{array}{l}\text { Realizar compresiones torácicas ininterrumpidas } \\
\text { durante al menos } 2 \text { minutos desde el inicio de la } \\
\text { llamada y posterior a la identificación del paro } \\
\text { cardiorespiratorio. }\end{array}$ \\
\hline
\end{tabular}

Manual del operador 9-1-1. Segundo proceso de capacitación para operadores telefónicos.

CNI: Centro Nacional de Información; SESNSP: Secretariado Ejecutivo del Sistema Nacional de Seguridad Pública.

de la emergencia, se realicen una serie de preguntas rápidas concretas y secuenciales con la finalidad de identificar en el menor tiempo posible la ausencia de signos de circulación y la necesidad de iniciar la RCP cuanto antes evitando el solicitar información adicional por medio de preguntas irrelevantes o gastar tiempo valioso tratando de averiguar si el paciente tiene o no respiración, tiempo que resulta determinante y muy valioso para asegurar una mejor supervivencia con el menor índice de secuelas neurológicas posibles (Fig. 3) ${ }^{10}$. 


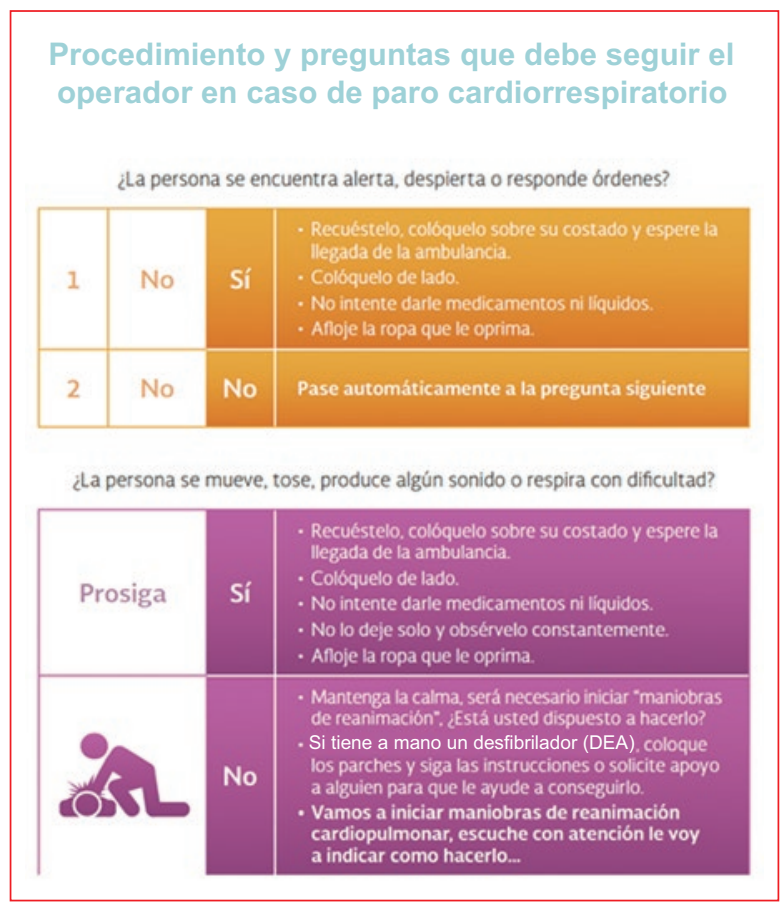

Figura 3. Algoritmo para la detección y atención temprana de pacientes en paro cardiorrespiratorio. Manual para Operadores Telefónicos 9-1-1. Segundo proceso de capacitación para operadores telefónicos. CNI: Centro Nacional de Información; SESNSP: Secretariado Ejecutivo del Sistema Nacional de Seguridad Pública.

Con la evolución de la medicina y el perfeccionamiento mundial de los sistemas de emergencia, acciones y herramientas como este protocolo médico resultan de suma importancia, ya que sin duda ayudarán a disminuir la mortalidad global por esta enfermedad y mejorar la calidad de vida de muchos mexicanos. Hasta la fecha de publicación de este artículo, gracias a la implementación y del uso de este protocolo ha sido posible evidenciar mediante el análisis nacional de llamadas una gran cantidad de casos con finales satisfactorios y verdaderas historias de éxito. Sin embargo, en opinión del autor es importante continuar trabajando en medidas que permitan perfeccionar este servicio, erradicar paradigmas y hacer un esfuerzo para educar más a la población sobre una cultura de actuación ante emergencias y de buen uso del número de emergencia 9-1-1 entre otras acciones como el desarrollo de sitios públicos cardioprotegidos que seguramente ayudarán a salvar cada día más vidas modificando el curso y pronóstico de esta grave enfermedad.

\section{Bibliografía}

1. Priori SG, Aliot E, Blomstrom-Lundqvist C, Bossaert L, Breithardt G, Brugada $P$, et al. Task force on sudden cardiac death of the European Society of Cardiology. Eur Heart J. 2001;22(16):1374-450.

2. Chugh SS, Reinier K, Teodorescu C, Evanado A, Kehr E, Al Samara M et al. Epidemiology of sudden cardiac death: clinical and research implications. Prog Cardiovasc Dis. 2008;51(3):213-28.

3. Lopera G, Curtis AB. Risk stratification for sudden cardiac death: current approaches and predictive value. Curr Cardiol Rev. 2009;5(1):56-64

4. Stecke EC, Reinier K, Marijon E, Narayanan K, Teodorescu C, Uy-Evanado A, et al. Public health burden of sudden cardiac death in the United States. Circ Arrhyth Electrophysiol. 2014;7(2):212-7.

5. Rodríguez-Reyes H, Muñoz-Gutiérrez M, Márquez MF, Pozas-Garza G, Asensio-Lafuente $E$, Ortíz-Galván $F$, et al. Muerte súbita cardíaca, estratificación del riesgo, prevención y tratamiento. Arch Cardiol Méx. 2015;85(4):329-36.

6. Adult Basic Life Support and Cardiopulmonary Resuscitation Quality Web-based Integrated 2010 \& 2015 American Heart Association Guidelines for Cardiopulmonary Resuscitation and Emergency Cardiovascular Care. Recuperado de https://eccguidelines.heart.org/index.php/circulation/cpr-ecc-guidelines-2/part-5-adult-basic-life-support-and-cardiopulmonary-resuscitation-quality/.

7. Kleinman ME, Goldberger ZD, Rea T, Swor RA, Bobrow BJ, Brennan EE, et al. 2017 American Heart Association Focused Update on Adult Basic Life Support and Cardiopulmonary Resuscitation Quality: An Update to the American Heart Association Guidelines for Cardiopulmonary Resuscitation and Emergency Cardiovascular Care. Circulation. 2018;137(1):e7-e13.

8. Vaillancourt C, Charette ML, Stiell IG, Wells GA. An evaluation of 9-1-1 calls to assess the effectiveness of dispatch-assisted cardiopulmonary resuscitation (CPR) instructions: design and methodology. BMC Emerg Med. 2008;8(5):12

9. Tanaka Y, Nishi T, Takase K, Yoshita Y, Wato Y, Taniguchi J, et al. Survey of a protocol to increase appropriate implementation of dispatcher-assisted cardiopulmonary resuscitation for out-of-hospital cardiac arrest. Circulation. 2014;129(17):1751-60.

10. Protocolo Médico Para la Atención del Paro Cardiorrespiratorio, Sánchez Arreola LD, Díaz Soto E, Manual de Capacitación para Operadores Telefónicos del Servicio 9-1-1, 3ra Edición, Centro Nacional de Información, 2018, pp. 1-54. 